NT@UW-03-07

\title{
Shapes of the Proton
}

\author{
Gerald A. Miller \\ University of Washington Seattle, WA 98195-1560
}

\begin{abstract}
A model proton wave function, constructed using Poincaré invariance, and constrained by recent electromagnetic form factor data, is used to study the shape of the proton. Spin-dependent quark densities are defined as matrix elements of density operators in proton states of definite spin-polarization, and shown to have an infinite variety of non-spherical shapes. For high momentum quarks with spin parallel to that of the proton, the shape resembles that of a peanut, but for quarks with anti-parallel spin the shape is that of a bagel.
\end{abstract}

Recent data [1,2] showing that the ratio of the proton's electric and magnetic form factor $G_{E} / G_{M}$, falls with increasing momentum transfer $Q^{2}$ for $1<Q^{2}<6 \mathrm{GeV}^{2}$ have created considerable attention. This behavior or the equivalent statement that the ratio of Pauli to Dirac form factors, $Q F_{2}\left(Q^{2}\right) / F_{1}\left(Q^{2}\right)$ is a approximately constant, indicates that quarks in the proton carry non-zero orbital angular momentum [3-6].

This note is concerned with the shape of the proton. Several subtleties and difficulties enter in determining the shape. As a particle of spin $1 / 2$, the proton can have no quadrupole moment, according to the Wigner-Eckhart theorem. Thus any non-spherical manifestation must reside in fluctuating components of the quantum wave function. Another difficulty occurs in using experiments in which momentum is transferred to the proton. The proton's final state carries different total momentum than that of the initial state, and the effects of boosts cause the initial and final state wave functions to differ. For example, one often thinks of a particle in relativistic motion having a pancake shape because of the effects of Lorentz contraction. Such an effect is caused by external influence and is therefore not a manifestation of the intrinsic shape of the proton. Another possible problem occurs through the use of light cone coordinates, which involves a separation of coordinates into longitudinal and transverse that complicates a simple interpretation. Our technique is to use a model of the proton wave function [7], [5], [8], constructed with Poincaré invariance and parameters constrained by data, to compute specific matrix elements using only the proton's rest frame wave function and ordinary coordinates.

The basic experimental observables concerning us are the form factors:

$$
F_{1}\left(Q^{2}\right)=\frac{1}{2 P^{+}}\left\langle N, \uparrow\left|J^{+}\right| N, \uparrow\right\rangle, \quad Q \kappa F_{2}\left(Q^{2}\right)=\frac{-2 M_{N}}{2 P^{+}}\left\langle N, \uparrow\left|J^{+}\right| N, \downarrow\right\rangle,
$$

with $F_{1}$ obtained from a non-spin flip matrix element, and $F_{2}$ from a spin-flip term. In evaluating the right-hand-side of Eq. (1) we take $J^{+}$to be that of free quarks [9], $\gamma^{+}$times the quark charge. We use a frame in which $q^{\nu}=\left(p^{\prime}-p\right)^{\nu}, Q^{2}=-q^{2}, q^{+}=0, \mathbf{q}=Q \mathbf{e}_{x}=-2 \mathbf{p}$.

Our three-quark wave function is constructed using symmetries $[11,12]$. It is antisymmetric, expressed in terms of relative momentum variables, an eigenstate of the spin-operator defined 
by the Pauli-Lubanski vector, is rotationally invariant and reduces to non-relativistic SU(6) wave function in the limit that the quark mass goes to infinity. The wave function is given by

$$
\Psi_{s}\left(p_{i}\right)=\Phi\left(M_{0}^{2}\right) u\left(p_{1}\right) u\left(p_{2}\right) u\left(p_{3}\right) \psi_{s}\left(p_{1}, p_{2}, p_{3}\right), \quad p_{i}=\mathbf{p}_{i} s_{i}, \tau_{i}
$$

where $\psi_{s}$ is a spin-isospin color amplitude factor [12], the $\mathbf{p}_{i}$ are expressed in terms of relative coordinates (with $-\mathbf{p}_{3 \perp}=\mathbf{K}$ ), the $u\left(p_{i}\right)$ are Dirac spinors and $\Phi$ is a spatial wave function. The arguments of the spatial wave function are taken as the mass-squared operator for a non-interacting system [13]:

$$
M_{3} \equiv 2\left(\mathbf{k}^{2}+m^{2}\right)^{1 / 2}, M_{0}=\left(\mathbf{K}^{2}+M_{3}^{2}\right)^{1 / 2}+\left(\mathbf{K}^{2}+m^{2}\right)^{1 / 2},
$$

where $\mathbf{k}$ is the relative momentum between quarks labeled 1 and 2 and $-\mathbf{K}$ is the momentum of the third quark [14]. The spatial wave function is of the Schlumpf [13] form:

$$
\Phi\left(M_{0}\right)=\frac{N}{\left(M_{0}^{2}+\beta^{2}\right)^{\gamma}}, \beta=0.607 \mathrm{GeV}, \gamma=3.5, m=0.267 \mathrm{GeV} .
$$

The value of $\gamma$ is chosen that $Q^{4} G_{M}\left(Q^{2}\right)$ is approximately constant for $Q^{2}>4 \mathrm{GeV}^{2}$. The values of $\beta$ and $m$ are determined by the charge radius and magnetic moment of the proton. Slightly different values of $\beta, \gamma$ and $m$ are used when the effects of the pion cloud are incorporated [8]. Using these newer values would cause very small differences here.

The calculation of electromagnetic form factor is completely defined once this wave function and operator $J^{+}$are specified. Although the evaluation was presented long ago [7] and explained recently [5], it is worthwhile to briefly explain how the constant nature of the ratio $Q F_{2} / F_{1}$ emerges from the relativistic nature of the calculation. The wave function Eq. (2) is completely anti-symmetric, so we may take $J^{+}$to act only on the third quark which absorbs the momentum of the virtual photon. The average charge of the third quark in the mixedsymmetric component of Eq. (2) vanishes, so the only component of the wave function that enters in the calculation of electromagnetic form factors is the mixed anti-symmetric component in which the first two quarks have a vanishing total angular momentum. Then the spin of the proton $s$ is governed by the third quark. The relevant Dirac spinor is:

$$
u\left(p_{3}=\mathbf{K}, s\right)=\frac{1}{\sqrt{E(K)+m}}\left(\begin{array}{c}
(E(K)+m)|s\rangle \\
\boldsymbol{\sigma} \cdot \mathbf{K}|s\rangle
\end{array}\right),
$$

with $E(K)=\left(K^{2}+m^{2}\right)^{1 / 2}$. The total angular momentum of the proton is denoted by $s$ and the lower component contains a term $\boldsymbol{\sigma} \cdot \mathbf{K}$ that allows the quark to have a spin opposite to that of the proton's total angular momentum. The vector $\mathbf{K}$ reveals the presence of the quark orbital angular momentum: the struck quark may carry a spin that is opposite to that of the proton. Consequently helicity is not conserved $[3,4,15]$.

Suppose $Q$ is very large compared to $\beta$ and the third quark changes its momentum from $\mathbf{K}$ to $\mathbf{K}^{\prime}$. Then the form factor depends on the matrix element of $\bar{u}\left(K^{\prime}, s^{\prime}\right) \gamma^{+} u(K, s)$. If we keep only the largest terms, those proportional to $Q$, we find 


$$
\bar{u}\left(K^{\prime}, s^{\prime}\right) \gamma^{+} u(K, s) \sim\left\langle s^{\prime}\left|Q+\frac{K^{+}}{M_{0}} Q i \sigma_{y}\right| s\right\rangle .
$$

The spin-flip term proportional to $i \sigma_{y}$ arises from the lower component of the spinor of Eq. (5), and this term has the same large factor $Q$ as the non-spin-flip term. Thus Eq. (1) tells us that $Q F_{2}$ and $F_{1}$ have the same dependence on $Q$, so their ratio is constant. The construction of an eigenstate of spin, mandates the use of Dirac spinors. The lower components of these spinors carry the quark orbital angular momentum responsible for the constant nature of $Q F_{2} / F_{1}$.

Our aim is to interpret these features of the wave function in terms of the shapes of the proton. The technique of defining spin-dependent density operators is introduced by presenting two simple examples. Consider a non-relativistic nuclear wave function consisting of a proton outside a $0^{+}$inert core. The proton is bound by a combination of a central and spin-orbit potential, and the single particle wave function is an eigenfunction of total angular momentum. Consider the case $(l, j s)=(1,1 / 2 s)$, with one unit of orbital angular momentum. Then the proton wave function is a two-component Pauli spinor: $\left\langle\mathbf{r}_{p} \mid \psi_{1,1 / 2 s}\right\rangle=R\left(r_{p}\right) \boldsymbol{\sigma} \cdot \hat{\mathbf{r}}_{p}|s\rangle$, where $\mathbf{r}_{p}$ is the proton's position, and $|s\rangle$ is a Pauli spinor. The charge density of this system is the expectation value of the density operator $\delta\left(\mathbf{r}-\mathbf{r}_{p}\right)$ : with $\rho(r)=\left\langle\psi_{1,1 / 2 s}\left|\delta\left(\mathbf{r}-\mathbf{r}_{p}\right)\right| \psi_{1,1 / 2 s}\right\rangle=$ $R^{2}(r)$, and is spherically symmetric. However, consider instead the case that we require the proton at a position $\mathbf{r}$ to have a spin in a direction defined by a unit vector $\mathbf{n}$. Then we define a spin-dependent density:

$$
\begin{array}{r}
\rho(\mathbf{r}, \mathbf{n})=\left\langle\psi_{1,1 / 2 s}\left|\delta\left(\mathbf{r}-\mathbf{r}_{p}\right) \frac{(1+\boldsymbol{\sigma} \cdot \mathbf{n})}{2}\right| \psi_{1,1 / 2 s}\right\rangle \\
=\frac{R^{2}(r)}{2}\langle s|1+2 \boldsymbol{\sigma} \cdot \hat{\mathbf{r}} \mathbf{n} \cdot \hat{\mathbf{r}}-\boldsymbol{\sigma} \cdot \mathbf{n}| s\rangle .
\end{array}
$$

An interesting special case is to take $\hat{\mathbf{n}}$ parallel or anti-parallel to the direction of the proton angular momentum s. The direction of this vector defines an axis (the "z-axis"), and the direction of vectors can be represented in terms of this axis: $\hat{\mathbf{s}} \cdot \hat{\mathbf{r}}=\cos \theta$. With this notation $\rho(\mathbf{r}, \mathbf{n}=\hat{\mathbf{s}})=R^{2}(r) \cos ^{2} \theta, \rho(\mathbf{r}, \mathbf{n}=-\hat{\mathbf{s}})=R^{2}(r) \sin ^{2} \theta$ and the non-spherical shape is exhibited clearly. Note that the average of these two cases would give a spherical shape (as would an average over the direction of the total angular momentum), but the ability to define a spin-dependent density allows the presence of the orbital angular momentum to be revealed in the shape of the computed density.

Another useful example is that of the Dirac electron wave function of the Hydrogen atom. This wave function is a four-component spinor given by $\left|\psi_{e}\right\rangle$ with

$$
\left\langle\mathbf{r}_{e} \mid \psi_{e}\right\rangle=N r_{e}^{\gamma} \exp \left(-m_{e} \alpha r_{e}\right)\left(\begin{array}{c}
1|s\rangle \\
i \alpha / 2 \boldsymbol{\sigma} \cdot \hat{\mathbf{r}}_{e}|s\rangle
\end{array}\right)
$$

where $\alpha$ is the fine structure constant and $\gamma=\sqrt{1-\alpha^{2}}$. We compute the expectation value of the spin-dependent density operator, in terms of Dirac matrices: $\left.\delta\left(\mathbf{r}-\mathbf{r}_{e}\right)\right)\left(1+\gamma^{0} \boldsymbol{\gamma} \cdot \mathbf{n} \gamma_{5}\right) / 2$, so that with $\mathbf{n}=\hat{\mathbf{s}}, \rho(\mathbf{r}, \hat{\mathbf{n}}=\hat{\mathbf{s}})=r^{-2 \gamma} \exp \left(-2 m_{e} \alpha r_{e}\right)\left[1+\alpha^{2} / 4 \cos ^{2} \theta\right]$. We see that the Hydrogen atom is deformed! The angular dependence is $\sim 1+10^{-5} \cos ^{2} \theta$ so that the shape 
is almost spherical. But the principle is clear: relativity as manifest by lower components of a Dirac wave function implies a deformed shape, if the matrix element is computed in a state of fixed total angular momentum. For $\mathbf{n}=-\hat{\mathbf{s}}, \rho(\mathbf{r}, \hat{\mathbf{n}}=-\hat{\mathbf{s}})=\alpha^{2} \sin ^{2} \theta / 4$.

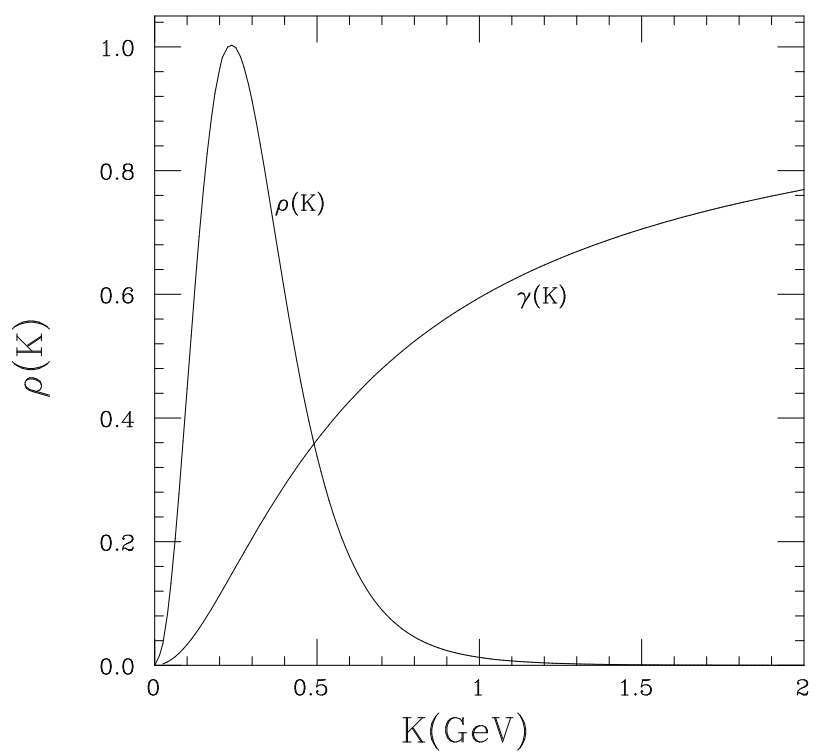

FIG. 1. Momentum probability $K^{2} \rho(K)$ in dimensionless units arbitrarily normalized.

With these examples in hand we turn to the proton. Its wave function is specified in momentum space, so we define a momentum-space, spin-dependent, charge-density operator:

$$
\hat{\rho}(\mathbf{K}, \mathbf{n})=\int \frac{d^{3} r}{(2 \pi)^{3}} e^{i \mathbf{K} \cdot \mathbf{r}} \bar{\psi}(\mathbf{r}) \frac{\widehat{Q}}{e}\left(\gamma^{0}+\boldsymbol{\gamma} \cdot \mathbf{n} \gamma_{5}\right) \psi(\mathbf{0}),
$$

where $\widehat{Q} / e$ is the quark charge operator in units of the proton charge. The quark field operators are evaluated at equal time. In QCD, one would need to add a factor between the field operators to ensure gauge invariance. With the present model, any gluons are contained within constituent quarks, so the factor becomes unity. For our model, it is convenient to use first-quantized notation so that:

$$
\hat{\rho}(\mathbf{K}, \mathbf{n}) \equiv \sum_{i=1,3}\left(Q_{i} / e\right) \delta\left(\mathbf{K}-\mathbf{K}_{i}\right)\left(1+\left(\gamma^{0} \boldsymbol{\gamma} \cdot \mathbf{n} \gamma_{5}\right)_{i}\right) / 2
$$

We may compute probabilities for a quark to have a momentum $\mathbf{K} \equiv(K, \theta, \phi)$ and spin direction $\mathbf{n}$, for a spin-polarized proton polarized in the $\hat{\mathbf{s}}$ direction. We find

$$
\rho(\mathbf{K}, \mathbf{n})=\left\langle\Psi_{s}|\hat{\rho}(\mathbf{K}, \hat{\mathbf{n}})| \Psi_{s}\right\rangle=\rho(K) \frac{1}{2}(1+\mathbf{n} \cdot \hat{\mathbf{s}}+\gamma(K)(1-\mathbf{n} \cdot \hat{\mathbf{s}}+2 \hat{\mathbf{K}} \cdot \mathbf{n} \hat{\mathbf{K}} \cdot \hat{\mathbf{s}}))
$$

with

$$
\rho(K) \equiv \int d^{3} k \Phi^{2}(k, K)(E(K)+m), \gamma(K) \equiv \frac{E(K)-m}{E(K)+m}
$$


Some special cases of Eq. (11) are interesting. Suppose the quark spin is parallel to the proton spin, $\mathbf{n}=\hat{\mathbf{s}}$, then $\hat{\rho}(K, \mathbf{n}=\hat{\mathbf{s}})=\rho(K)\left(1+\gamma(K) \cos ^{2} \theta\right)$. For small $K$ the shape is nearly spherical, but for large $K$ the $\cos ^{2} \theta$ term becomes prominent. On the other hand, the quark spin could be anti-parallel to the proton spin, $\mathbf{n}=-\hat{\mathbf{s}}$. Then we find: $\hat{\rho}(K, \mathbf{n}=$ $-\hat{\mathbf{s}})=\rho(K) \gamma(K) \sin ^{2} \theta$, and the shape is that of a torus. We may also take the quark spin perpendicular to the proton spin $\mathbf{n} \cdot \mathbf{s}=0$, so that $\rho(\mathbf{K}, \mathbf{n} \cdot \mathbf{s}=0)=\rho(K)(1+\gamma(K)) / 2+$ $\gamma(K) \sin \theta \cos \theta\left(\cos \phi n_{x}+\sin \phi n_{y}\right)$, to display the dependence on the azimuthal angle. In each case, the non-spherical nature arises from the term proportional to $\gamma(K)$ caused by the lower components of the Dirac spinor (5).
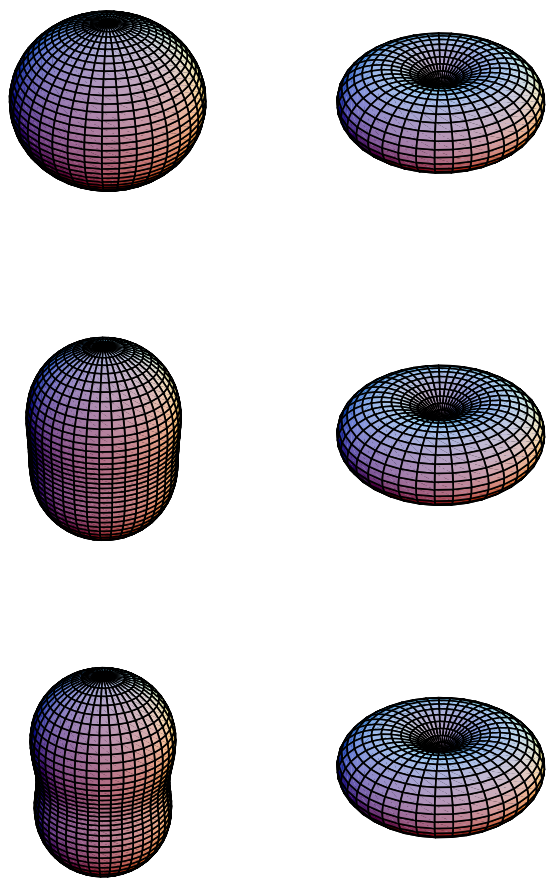

FIG. 2. Shapes of the proton. $\mathbf{s}$ is in the vertical direction $\hat{\mathbf{z}}$. Left column quark spin parallel to nucleon $\operatorname{spin} \mathbf{n}=\hat{\mathbf{s}}$. Right column : quark spin anti-parallel to nucleon $\operatorname{spin} \mathbf{n}=-\mathbf{s}$. The value of $K$ increases from 0 to 1 to $4 \mathrm{GeV} / \mathrm{c}$.

We turn to the numerical evaluation and display of these shapes. The shape for a given value of $K$ is determined by the ratio $\gamma(K)$ which reaches a value of 0.6 for $K=1 \mathrm{GeV} / \mathrm{c}$. This implies considerable non-sphericity. The probability that a given value of $K$ is reached is determined by the function $K^{2} \rho(K)$, displayed in Fig. 1 . The most likely value of $K$ corresponds to $\gamma(K)=0.16$, and $\gamma(K) \leq 1$.

The shapes for the cases of quark spin parallel and anti-parallel to the polarization direction 
of the proton $\mathbf{s}$ are displayed in Fig. 2. As the value of $K$ increases from 0 to $4 \mathrm{GeV} / \mathrm{c}$ the shape varies from that of a sphere to that of a peanut, if $\mathbf{n} \| \mathbf{s}$. The torus or bagel shape is obtained if $-\mathbf{n} \| \mathbf{s}$. Taking $\mathbf{n} \perp \mathbf{s}$ leads to some very unusual shapes shown in Fig. 3 .
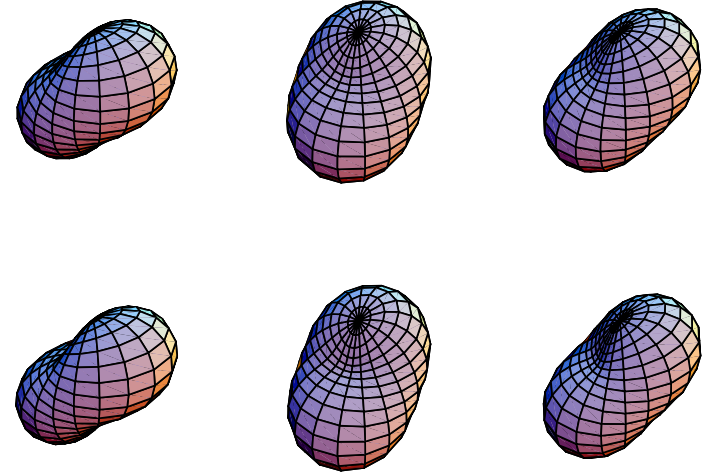

FIG. 3. $\mathbf{n} \cdot \mathbf{s}=0$. Left column, $\mathbf{n}=\hat{\mathbf{x}}$ (out of page), central: $\mathbf{n}=\hat{\mathbf{y}}$, right $\mathbf{n}=(\hat{\mathbf{x}}+\hat{\mathbf{y}}) / \sqrt{2}$. The momentum $K$ increases from 1 to $4 \mathrm{GeV} / \mathrm{c}$.

It is worthwhile to obtain a coordinate space look at the shape of the proton. One can determine the probability for the separation of a quark from the center of mass to be $\mathbf{r}$ because $\mathbf{r}$ is canonically conjugate to $\mathbf{K}$. We define a coordinate-space, spin-dependent, charge-density operator that acts on the quarks in the cm frame:

$$
\hat{\rho}(\mathbf{r}, \mathbf{n}) \equiv \sum_{i=1,3}\left(Q_{i} / e\right) \delta\left(\mathbf{r}-\mathbf{r}_{i}\right)\left(1+\left(\gamma^{0} \boldsymbol{\gamma} \cdot \mathbf{n} \gamma_{5}\right)_{i}\right) / 2
$$

so that we may compute probabilities for a quark to have a position $\mathbf{r} \equiv(r, \theta, \phi)$ and spin direction $\mathbf{n}$. We find

$$
\rho(\mathbf{r}, \mathbf{n})=\left\langle\Psi_{s}|\hat{\rho}(\mathbf{r}, \mathbf{n})| \Psi_{s}\right\rangle=\int d^{3} k \chi^{\dagger}(k, \mathbf{r}) \frac{1}{2}\left(1+\gamma^{0} \boldsymbol{\gamma} \cdot \mathbf{n} \gamma_{5}\right) \chi(k, \mathbf{r}),
$$

with

$$
\chi(k, \mathbf{r})=\left(\begin{array}{c}
F_{k}(r)|s\rangle \\
-i \boldsymbol{\sigma} \cdot \hat{\mathbf{r}} G_{k}(r)|s\rangle
\end{array}\right)
$$

and $F_{k}(r)=\int d^{3} K \Phi(k, K)(E(K)+M)^{1 / 2} e^{i \mathbf{K} \cdot \mathbf{r}}, G_{k}(r)=\frac{\partial}{\partial r} \int d^{3} K \Phi(k, K) \frac{e^{i \mathbf{K} \cdot \mathbf{r}}}{(E(K)+M)^{1 / 2}}$. We find

$$
\rho(\mathbf{r}, \mathbf{n})=\rho_{U}(r) \frac{1}{2}(1+\mathbf{n} \cdot \hat{\mathbf{s}})+\rho_{L}(r) \frac{1}{2}(1+2 \hat{\mathbf{r}} \cdot \hat{\mathbf{s}} \mathbf{n} \cdot \hat{\mathbf{r}}-\mathbf{n} \cdot \hat{\mathbf{s}}),
$$

where $\rho_{U}(r) \equiv \int d^{3} k F_{k}^{2}(r), \rho_{L}(r) \equiv \int d^{3} k G_{k}^{2}(r)$. The pattern is similar to the one in momentum space, with $\rho(\mathbf{r}, \mathbf{n}=\hat{\mathbf{s}})=\rho_{U}(r)+\rho_{L}(r) \cos ^{2} \theta, \quad \rho(\mathbf{r}, \mathbf{n}=-\hat{\mathbf{s}})=\rho_{L}(r) \sin ^{2} \theta$, and $\rho\left(\mathbf{r}, \mathbf{n}=\frac{\hat{\mathbf{x}}+\hat{\mathbf{y}}}{\sqrt{2}}\right)=\frac{1}{2} \rho_{U}(r)+\frac{1}{2} \rho_{L}(r)\left(1+\frac{2}{\sqrt{2}} \cos \theta \sin \theta(\cos \phi+\sin \phi)\right)$. 


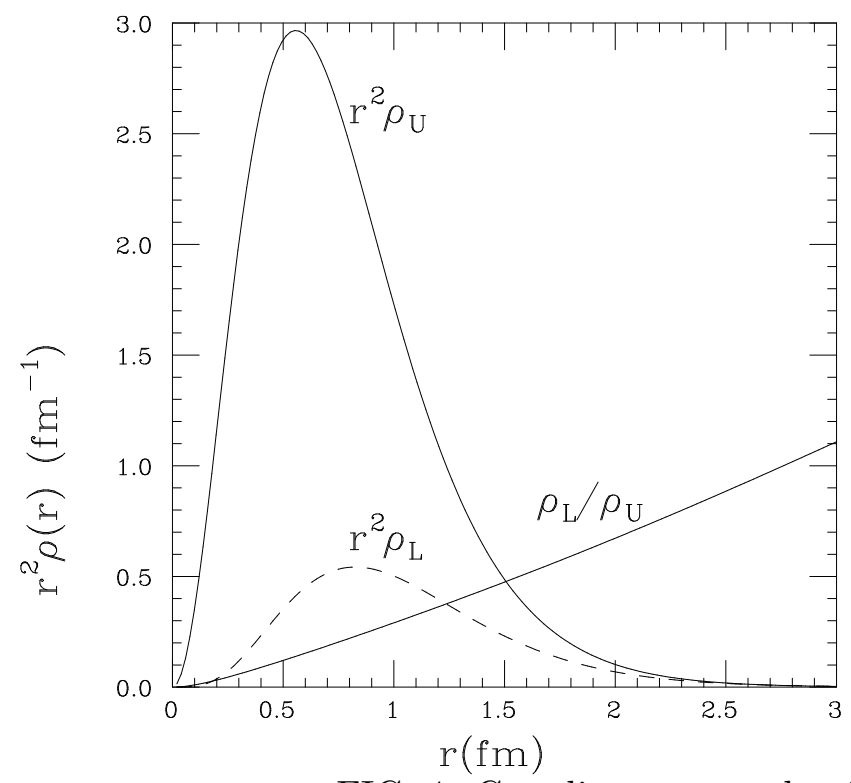

FIG. 4. Coordinate space densities

The ratio $\rho_{L} / \rho_{U}$ controls the relativistic effects. This can be much larger (Fig. 4) than the factor $\gamma(K)$ (limited by unity) controlling the momentum-space shapes, so more extreme shapes are possible. The most likely value of $\rho_{L} / \rho_{U}$ is about 0.25 , but there is no limit. The case with $\rho_{L} / \rho_{U}=3$ is shown in in Fig. 5 . A pretzel form is obtained if $\mathbf{n}$ is out of the page.
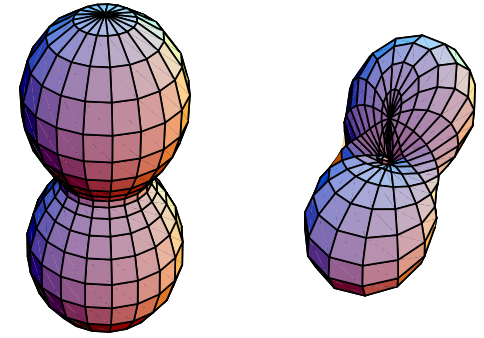

FIG. 5. Shape of the proton coordinate space. Left: $\mathbf{n}=\mathbf{s}$, right: $\mathbf{n}$ points out of the page

The shape of the proton may be defined in terms of matrix elements of spin-dependent density operators Eqs. $(10,13)$ taken for protons in any fixed polarization state. Relativity mandates the use of Dirac spinors to describe the quarks. These components, embodied in Eq. (6), lead to a constant ratio of $Q F_{2} / F_{1}$ in accord with observation, and also to shapes of an infinite variety, Eqs. (11,16), Figs. 2,3,5.

We next consider experiments capable of measuring the matrix element $\hat{\rho}(\mathbf{K}, \mathbf{n})$, Eq. (9), for real nucleons $|N\rangle$. Observe that $\int d^{3} K \hat{\rho}(\mathbf{K}, \mathbf{n})$ is a local operator. Its matrix element is a linear combination of the charge, integrals of spin-dependent structure functions $\Delta q$, and $g_{A}$, it can be determined from previous measurements. We find

$$
\int d^{3} K\langle N|\hat{\rho}(\mathbf{K}, \mathbf{n}= \pm \hat{\mathbf{s}})| N\rangle=\frac{1}{2}\left\langle N\left|\bar{\psi}(0) \frac{\hat{Q}}{e}\left(\gamma^{0} \pm \gamma^{3} \gamma^{5}\right) \psi(0)\right| N\right\rangle
$$




$$
=\frac{1}{2}\left(1 \pm\left(\frac{1}{6}(\Delta u+\Delta d+\Delta s)+\frac{1}{2} g_{A}\right)=0.5 \pm 0.34\right.
$$

in which numerical values of $\Delta q$ are taken from Ref. [16]. The model we use gives $0.5 \pm 0.37$ for the above quantity, indicating that the results shown here may not be unrealistic.

The task of determining $\rho(\mathbf{K}, \mathbf{n})$ as a function of $\mathbf{K}$ remains open. However, the specific relativistic effects of orbital angular momentum and the related spin-flip effects responsible for the non-spherical shapes can be expected to influence many measurable quantities. These include: spin-dependent structure functions $g_{1}, g_{2}$, the $N \Delta$ electromagnetic transition form factor, and spin-dependent cross sections in high momentum transfer scattering of polarized protons. These topics will be addressed in other publications.

\section{ACKNOWLEDGMENTS}

I thank the USDOE for partial support of this work. I thank M. Burkardt for useful discussions, and C. Glasshauser and J. Ralston for emphasizing the importance of understanding the shape of the proton.

[1] M. K. Jones et al. [Jefferson Lab Hall A Collaboration], Phys. Rev. Lett. 84, 1398 (2000)

[2] O. Gayouet al.Phys. Rev. Lett. 88, 092301 (2002)

[3] P. Jain, B. Pire and J. P. Ralston, Phys. Rept. 271, 67 (1996); T. Gousset, B. Pire and J. P. Ralston, Phys. Rev. D 53, 1202 (1996)

[4] V. M. Braun, A. Lenz, N. Mahnke and E. Stein, Phys. Rev. D 65, 074011 (2002).

[5] G. A. Miller and M. R. Frank, Phys. Rev. C 65, 065205 (2002)

[6] A. V. Belitsky, X-d. Ji and F. Yuan, arXiv:hep-ph/0212351.

[7] M.R. Frank, B.K. Jennings and G.A. Miller, Phys. Rev. C 54, 920 (1996).

[8] G. A. Miller, Phys. Rev. C 66, 032201 (2002) [arXiv:nucl-th/0207007].

[9] When the pion cloud [10] is included [8], $J^{+}$also includes the current of free pions, which are important at low values of $Q^{2}$.

[10] S. Théberge, A. W. Thomas and G. A. Miller, Phys. Rev. D22 (1980) 2838; (1981) 2106; A. W. Thomas, S. Théberge, and G. A. Miller, Phys. Rev. D24 (1981) 216; S. Théberge, G. A. Miller and A. W. Thomas, Can. J. Phys. 60, 59 (1982). G. A. Miller, A. W. Thomas and S. Théberge, Phys. Lett. B 91, 192 (1980).

[11] V. B. Berestetskii and M. V. Terent'ev. Sov. J. Nucl. Phys. 25, 347 (1977).

[12] P. L. Chung and F. Coester. Phys. Rev. D 44, 229, (1991); F. Coester, Prog. in Part. and Nucl. Phys. 29, 1 (1992).

[13] F. Schlumpf, U. Zurich Ph. D. Thesis, hep-ph/9211255.

[14] Calculations of the form factors are simplified by using light front dynamics to include the effects of the boost. For our model the difference between the light front and instant forms is contained 
in a coordinate transformation, which can be made or not depending on the application. Here we will use $\mathbf{q}=0$, so we use the instant form.

[15] The ratio of $Q F_{2} / F_{1}$ is a ratio of a helicity-non-conserving to a helicity conserving matrix element, if the reference frame of Eq. (1) is used.

[16] A. W. Thomas and W. Weise, "The Structure Of The Nucleon,", Wiley (Berlin 2001) 